\title{
High-Speed impact experiment for evaluation of magnetorheological fluid's shock-absorption performance
}

\author{
Yuya Mitani ${ }^{1}$, Takahiro Yano $^{2}$, Takuyoh Hagi ${ }^{2}$, Keiko Watanabe ${ }^{1, *}$, and Koji Fukudome ${ }^{1}$ \\ ${ }^{1}$ Ritsumeikan University, Department of Mechanical Engineering, 1-1-1 Noji-higashi, Kusatsu, Shiga 525-8577, Japan \\ ${ }^{2}$ Ritsumeikan University, Department of Advanced Mechanical Engineering and Robotics, 1-1-1 Noji-higashi, Kusatsu, \\ Shiga 525-8577, Japan
}

\begin{abstract}
Magnetorheological (MR) fluids are categorized as smart fluids, which are made of small iron particles suspended in carrier fluids such as silicone oil. The presence of a magnetic field will instantaneously increase the viscosity of the MR fluid, also known as the MR effect. The application of the MR fluid as viscous dampers to automobiles and buildings has shown excellent performance in shock absorption. To expand the practical application of the MR fluid, various evaluations of shock-absorption performance under high-loading conditions are needed. Therefore, we decided to investigate its performance in high-speed impact conditions. Impact experiments were conducted in different liquids - tap water, two types of silicone oils with different kinematic viscosities, and an MR fluid - and it was investigated how the properties of each liquid affect the shock-absorption performance. Accordingly, it was found that kinematic viscosity and compressibility affect shock-absorption performance. The kinematic viscosity did not affect the speed attenuation of the projectile. Furthermore, it was found that the compressibility affected the pressure wave generated by the entry of a projectile into the liquid.
\end{abstract}

\section{Introduction}

Magnetorheological (MR) fluids are categorized as smart fluids, which consist of iron particles (ferromagnetic particles with a diameter of several micrometers), solvent (hydrocarbon oil and silicone oil), and surfactant for improving the dispersibility of particles. When a magnetic field is not applied, an MR fluid shows the characteristics of a Newtonian fluid. On application of an external magnetic field, iron particles in the fluid are reversibly magnetized in several milliseconds and the flow characteristic changes to that of a Bingham fluid. In other words, the viscosity rapidly increases, and this property changes the fluid into a semisolid. It has already been used as a shock-absorbing device for automobile dampers, seismic isolation device, and impact absorbers by utilizing this feature. Therefore, it is believed that the application of MR fluids will increase in the future. For example, application to the case of high loads, such as an airplane's landing gears, is considered. In previous studies, to understand the dynamic properties of MR fluids, the increase in yield shear stress due to magnetic field applied by Carlson et al. [1], and result of quasi-static compression examination by Mazlan et al. [2] have been reported. There are many fundamental studies for such cases. However, vibration and shock suppression are only individually implemented and examined for each application. Therefore, we decided to evaluate the shockabsorption performance when a high-speed projectile impacts the MR fluid. Two types of shock-absorption performance are conceivable when the high-speed projectile enters the liquid; one is the deceleration of the penetrative projectile and the other is the attenuation of the pressure wave generated by projectile impact. As the first step, we conducted experiments on tap water and silicone oil used as a solvent for the MR fluid to understand how the high-speed projectile moving through the fluid decelerates and how the pressure wave propagating through the fluid attenuates. In the next step, we conducted experiments on MR fluids to understand the attenuation of the pressure wave. We compared MR fluids with other liquids, and investigated factors that affect shock-absorption performance.

\section{High-speed impact and penetration experiment}

In this experiment, considering the target as a liquid, a vertical gas gun is used as a projectile accelerator, and the projectile impacts the liquid at a speed of $220 \mathrm{~m} / \mathrm{s}$. The shape of the projectile is shown in Fig. 1. It consists of an impactor (brass) and a sabot (high-density polyethylene), and its weight is about $16 \mathrm{~g}$. A neodymium magnet ( $4 \mathrm{~mm}$ in diameter and $4 \mathrm{~mm}$ in height) is mounted on the back of the sabot to measure the impact speed of the projectile in experiments on tap water and silicone oil. The induced electromotive force generated when the magnet passes through the interior of an in-house-fabricated velocimeter having three coils arranged at intervals of $20 \mathrm{~mm}$ is acquired. The impact speed of the projectile is calculated by using the time difference of outputs and the distance between the coils.

\footnotetext{
Corresponding author: keikow@,fc.ritsumei.ac.jp
} 
The experiment on MR fluids in the absence of a magnetic field cannot be used to measure the speed in the presence of magnets because of the property change of the MR fluid caused by the magnet. Therefore, by using a laser, the times when the projectile obstructed and cut off the laser were measured, and the impact speed of the projectile was calculated by using the obstruction time of the laser and the total length of the projectile. Tap water, low-viscosity silicone oil, highviscosity silicone oil, and MR fluid were used as the target liquids. The MR fluid used in the experiment consisted of 66.5 vol\% high-viscosity silicone oil, 30 vol\% carbonyl iron particles, and 3.5 vol $\%$ surfactant. The sound speed in an MR fluid in the absence of a magnetic field is calculated by using Wood's effective medium theory [3]. The relationship between the sound speed, average volume density, and average bulk modulus of the suspension is formulated by Wood's effective medium theory, and the sound speed, when no magnetic field is applied to the suspension, can be predicted.

$$
\begin{gathered}
v=\sqrt{\frac{\beta}{\rho}} \\
\rho=\varphi \rho_{\mathrm{S}}+(1-\varphi) \rho_{\mathrm{L}} \\
\beta^{-1}=\varphi \beta_{\mathrm{S}}^{-1}+(1-\varphi) \beta_{\mathrm{L}}^{-1}
\end{gathered}
$$

where $v, \rho, \beta$, and $\varphi$ are the sound speed in the medium, average volume density, average bulk modulus of the medium, and volume fraction, respectively. Further, the subscripts $\mathrm{S}$ and $\mathrm{L}$ stand for solid and liquid, respectively. The physical properties of each liquid are listed in Table 1. The MR fluid's physical properties are obtained by calculation and measurement. The other values are published values. The kinematic viscosity of the MR fluid is not consistent and depends on the shear rate. In the interior of the experimental container, three sensors (Müller-Platte Needle Probe, Dr. Müller Instruments) were installed at $30-\mathrm{mm}$ intervals to measure the pressure wave generated by the projectile colliding with the liquid. The details of the sensor installation are shown in Fig. 2. The sensor needs to be installed perpendicular to the pressure wave. The propagation of the pressure wave is reproduced by numerical analysis, and an appropriate angle for each sensor position is determined. The penetration speed is calculated by impact experiments, which are conducted in transparent liquids.

\section{Experimental results and discussion}

\subsection{History of projectile speed}

The relationship between projectile speed and depth in the liquid is shown in Fig. 3. In the high-speed impact and penetration experiments on tap water, low-viscosity silicone oil, and high-viscosity silicone oil, the deceleration became almost constant and abrupt deceleration was not observed. Since the liquid provides viscous resistance to the sides of the projectile during penetration, it was assumed that the kinematic viscosity of the liquid affects the attenuation of the projectile speed. In spite of kinematic viscosity change, the attenuation of the projectile speed was not affected by the impact of the projectile against the liquid surface to a depth of $100 \mathrm{~mm}$. A cavity is formed around the projectile, and the liquid does not directly contact the sides of the projectile because the projectile penetrates the liquid at high speed. Therefore, it is assumed that only the viscous resistance of air inside the cavity acts on the sides of the projectile.

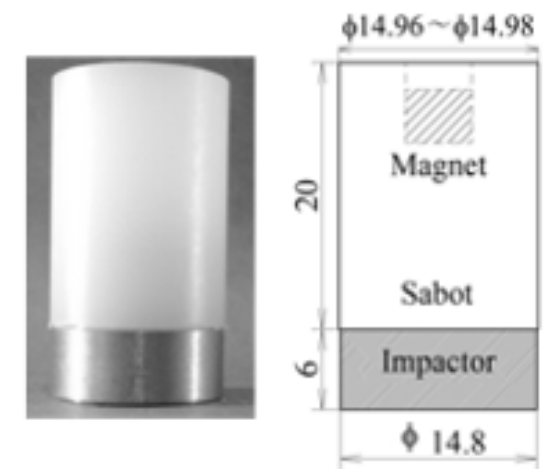

Unit:-mm

Fig. 1. Projectile.

Table 1. Physical properties of each liquid.

\begin{tabular}{cccc}
\hline & $\begin{array}{c}\text { Kinematic } \\
\text { viscosity } \\
{\left[\mathrm{m}^{2} / \mathrm{s}\right]\left(\text { at } 25^{\circ}\right)}\end{array}$ & $\begin{array}{c}\text { Sound } \\
\text { speed } \\
{[\mathrm{m} / \mathrm{s}]}\end{array}$ & $\begin{array}{c}\text { Density } \\
{\left[\mathrm{kg} / \mathrm{m}^{3}\right]}\end{array}$ \\
\hline $\begin{array}{c}\text { Tap water } \\
\text { Low-viscosity } \\
\text { silicone oil }\end{array}$ & $0.9 \times 10^{-6}$ & 1509 & 997 \\
$\begin{array}{c}\text { High-viscosity } \\
\text { silicone oil } \\
\text { MR fluid }\end{array}$ & $100 \times 10^{-6}$ & 901.3 & 818 \\
\hline
\end{tabular}

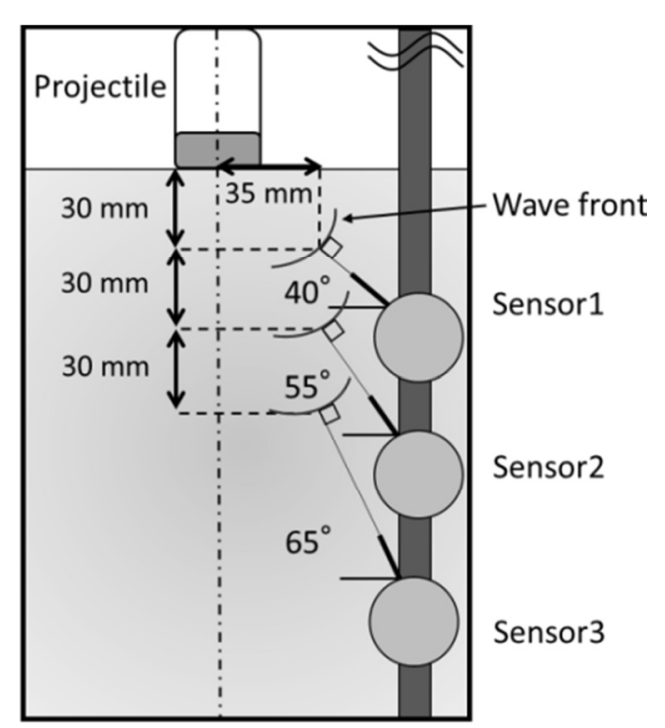

Fig. 2. Schematic diagram of probe configuration. 


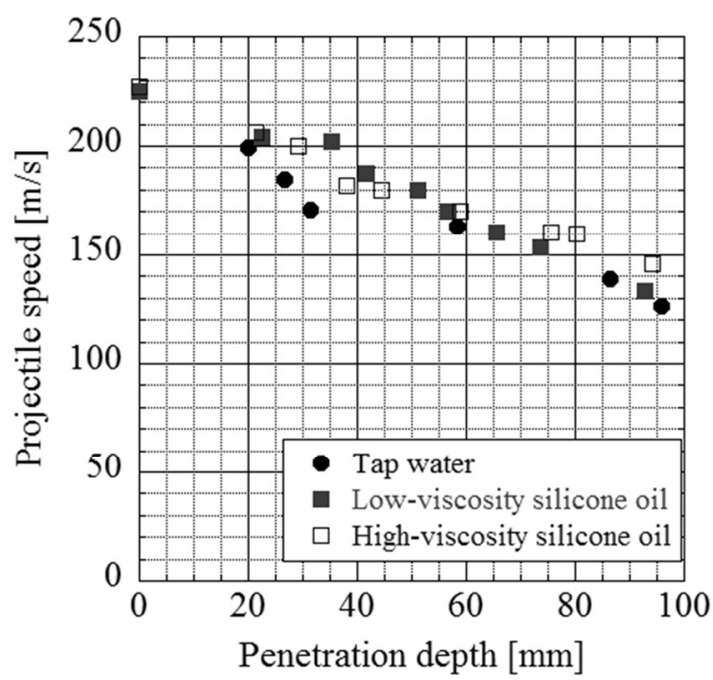

Fig. 3. Speed profile of projectile in tap water, low-viscosity, and high-viscosity silicone oil.

\subsection{Pressure-wave-induced impact}

The results of detection of the pressure wave generated by the projectile impact the liquids are shown in Figs. 47. The time at which the projectile impacts the liquid surface is shown as $0 \mu \mathrm{s}$ in the figure. In all the experiments, it can be confirmed that the peak pressure is attenuated from sensor 1 to sensor 3 . The time at which peak pressure is detected is the time of pressure wave arrival at the tip of the sensor. The peak pressure of tap water is the highest at any sensor position, and comparing the silicone oils with different kinematic viscosities, the peak pressure is almost the same at any sensor position. Therefore, pressure wave attenuation does not depend on kinematic viscosity, and we expect it to depend on the physical property of the liquid. Since the front side of the projectile compresses the anterior of the liquid, we focused on the compressibility of the liquid. The relationship between compressibility $\alpha$, sound speed $c$, and density $\rho$ is given by equation (4).

$$
\alpha=\frac{1}{c^{2} \rho}
$$

Equation (5) establishes the relation between compressibility $\alpha$ and pressure.

$$
\Delta p=\frac{1}{\alpha} \frac{\Delta V}{V}
$$

where $\Delta p$ is the pressure rise, $\Delta V$ is the volumetric change after compression, and $V$ is the volume before compression. From the calculations of the compressibility from the density and sound speed of each liquid, it is found that for water it is approximately $0.44 \times 10^{-3}[1 / \mathrm{MPa}]$, for low-viscosity silicone oil it is approximately $1.50 \times 10^{-3} \quad[1 / \mathrm{MPa}]$, for high-viscosity silicone oil it is approximately $1.07 \times 10^{-3}[1 / \mathrm{MPa}]$, and for the MR fluid it is approximately $0.74 \times 10^{-3}[1 / \mathrm{MPa}]$. Therefore, the compressibility of water is small and the pressure acting on the fluid is the highest when comparing water with other fluids. It is consistent with the experimental results. Since the compressibility of the MR fluid is larger than that of tap water and smaller than that of silicone oils, the pressure acting on the fluid is smaller than that of tap water and larger than that of silicone oils. It also agrees with the experimental results of sensor 1 . We conclude that the compressibility affects peak pressure.

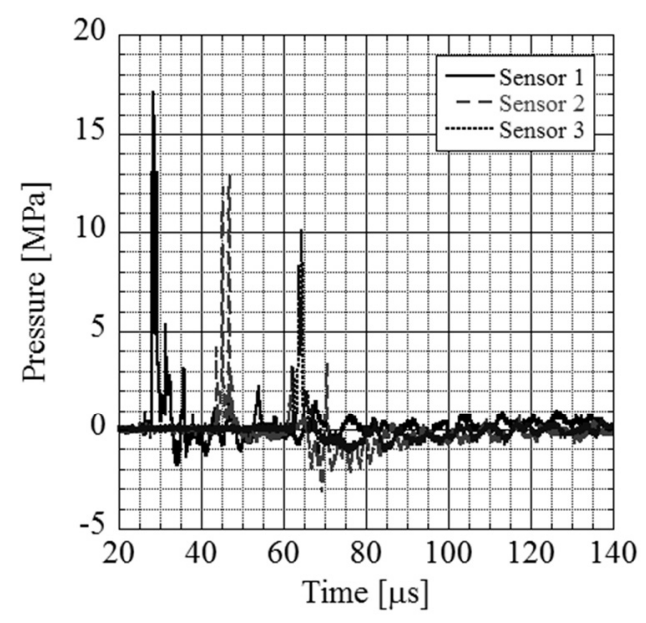

Fig. 4. Pressure wave signals in tap water.

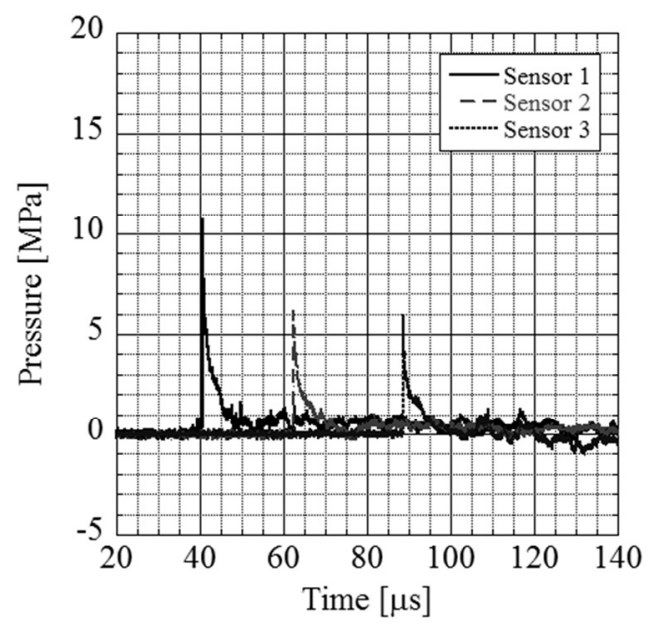

Fig. 5. Pressure wave signals in low-viscosity silicone oil.

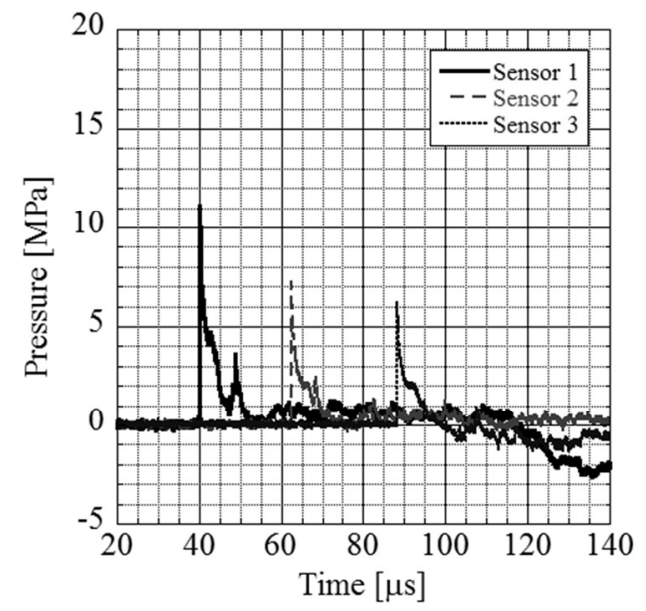

Fig. 6. Pressure wave signals in high-viscosity silicone oil. 


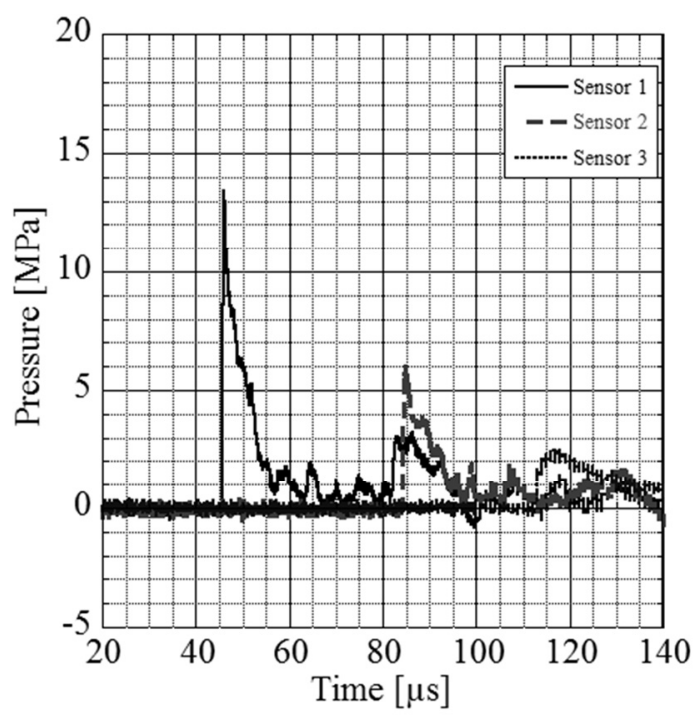

Fig. 7. Pressure wave signals in MR fluid.

\subsection{Peak pressure}

The sensor positions for measuring peak pressure are shown in Fig. 8. The pressure on each liquid is measured experimentally with sensors installed at the circular positions shown in Fig. 8. However, to investigate the attenuation of the spherical pressure wave caused by the impact of the projectile on the liquid, it must be evaluated on the extension line of the sensor installed at $40^{\circ}$. Therefore, we correct the peak pressures of sensor 2 and sensor 3 by using equation (6). It is a correction formula from the experiment conducted by Huang et al. [4] for the maximum value of a pressure wave propagating in the circumferential direction.

$$
P=P_{90} \sin (\theta \pm 7)
$$

where $P$ is the measured pressure, $P_{90}$ is the peak pressure of the spherical pressure wave, and $\theta$ is the angle that determines the position of the pressure wave in the circumferential direction. The value of $\pm 7^{\circ}$ in the correction formula is defined as the range of the error bar. The relationship between peak pressure and distance from the impact point is shown in Fig. 10. Experimental results on all liquids show that the peak pressure is most attenuated between sensor 1 and sensor 2 . The pressure attenuation continues between sensor 2 and sensor 3 in the results of the MR fluid, whereas the pressure attenuation effect is small in the results of tap water and silicone oils. We normalized the values of sensor 2 and sensor 3 based on the pressure of sensor 1 to investigate the influence of propagation distance on the attenuation of the pressure wave, and comparing the normalized values with the function $1 / r$ ( $r$ is the wave propagation distance) that affects the attenuation of the spherical wave. These results are shown in Figs. 11-14. In the experimental result for tap water, it can confirmed that pressure value attenuates with a tendency close to $1 / r$, and it is dominated by the attenuation effect from the propagation distance. For the silicone oils, the normalized pressure of sensor 2 deviates significantly from the $1 / r$ function than the result for water. In addition, in the MR fluid, the normalized pressure of sensor 2 and sensor 3 has the same trend. Therefore, the maximum value of the pressure wave is affected by the attenuation effect, which depends on the propagation distance. In addition, we considered it to be attenuated by the effect of physical properties such as kinematic viscosity and sound speed. By comparing the results for tap water and low-viscosity silicone oil, it was found that sound speed affects the pressure wave. Furthermore, it was found from the result that density and kinematic viscosity affect the pressure wave in the MR fluid.

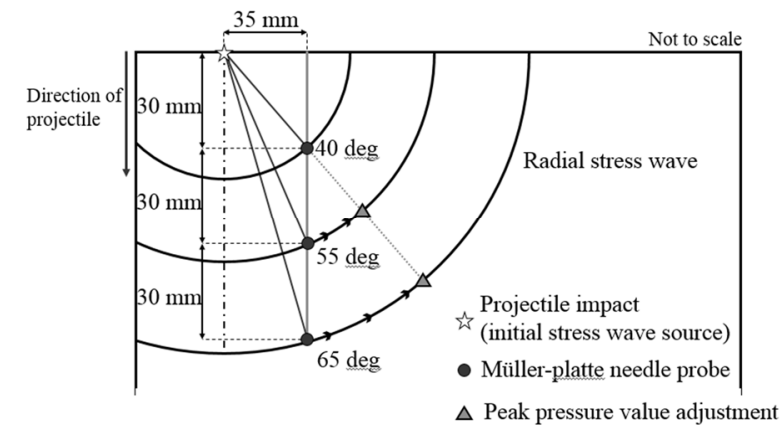

Fig. 8. Schematic diagram for peak pressure value.

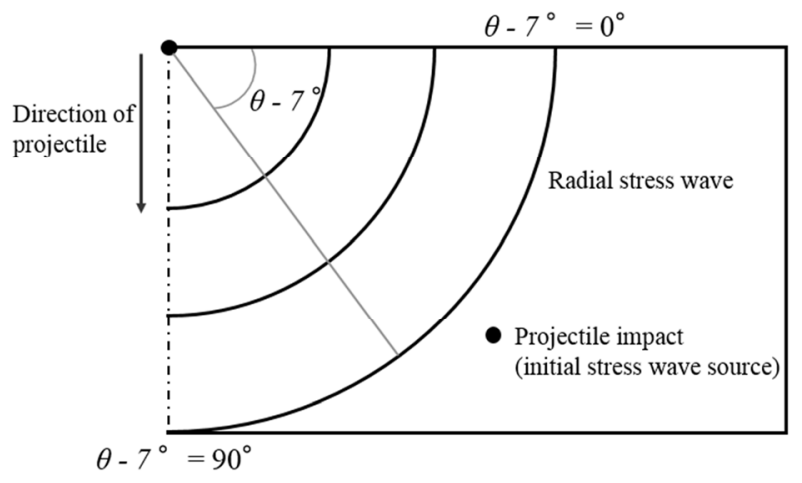

Fig. 9. Relationship between circumference angle and pressure.

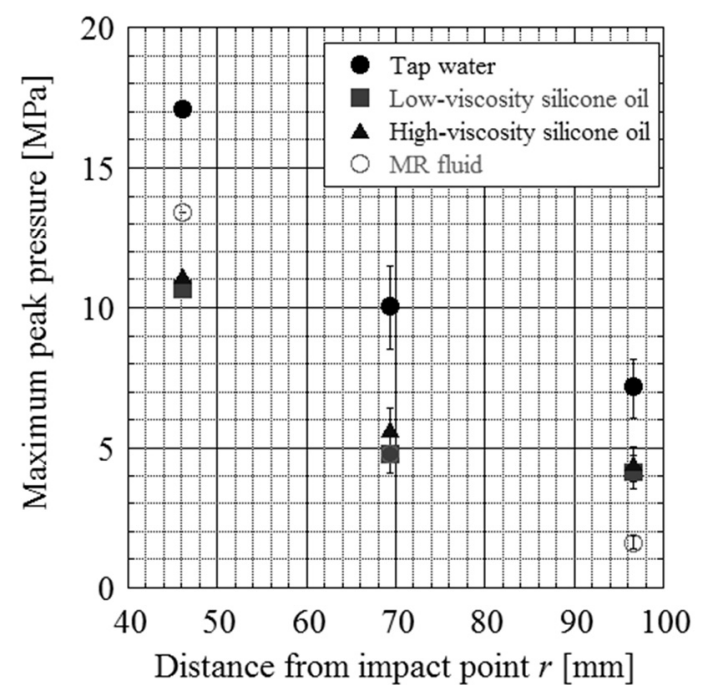

Fig. 10. Schematic diagram of peak-pressure adjustment value. 


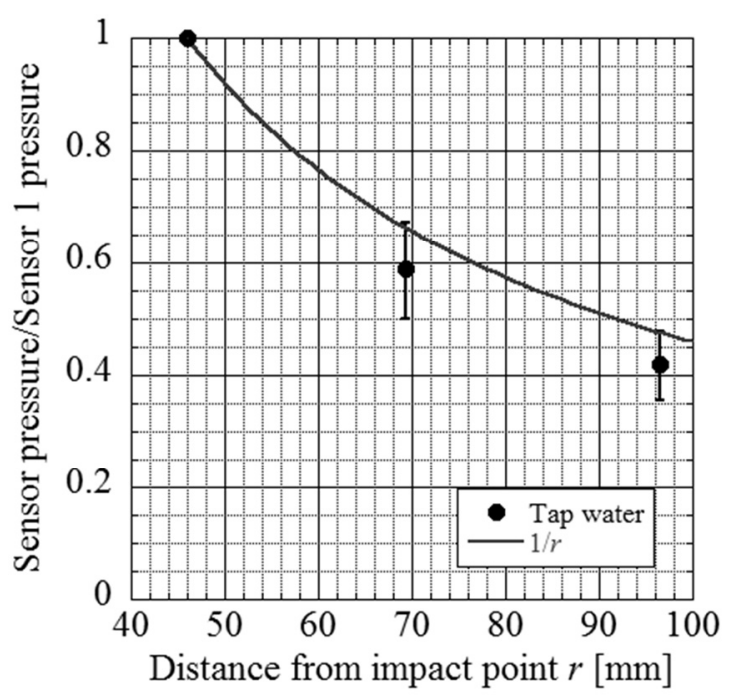

Fig. 11. Comparison between normalized peak pressure for tap water and $1 / r$ curve.

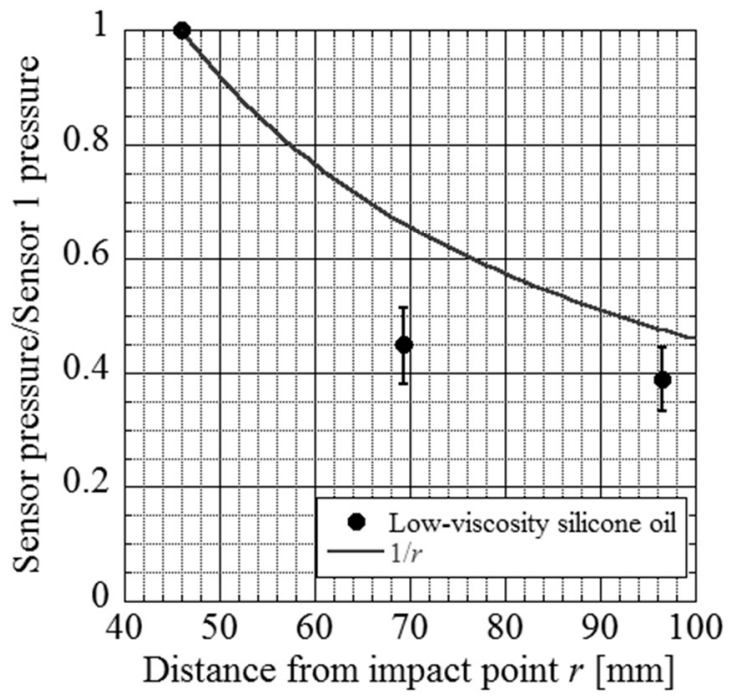

Fig. 12. Comparison between normalized peak pressure for low-viscosity silicone oil and $1 / r$ curve.

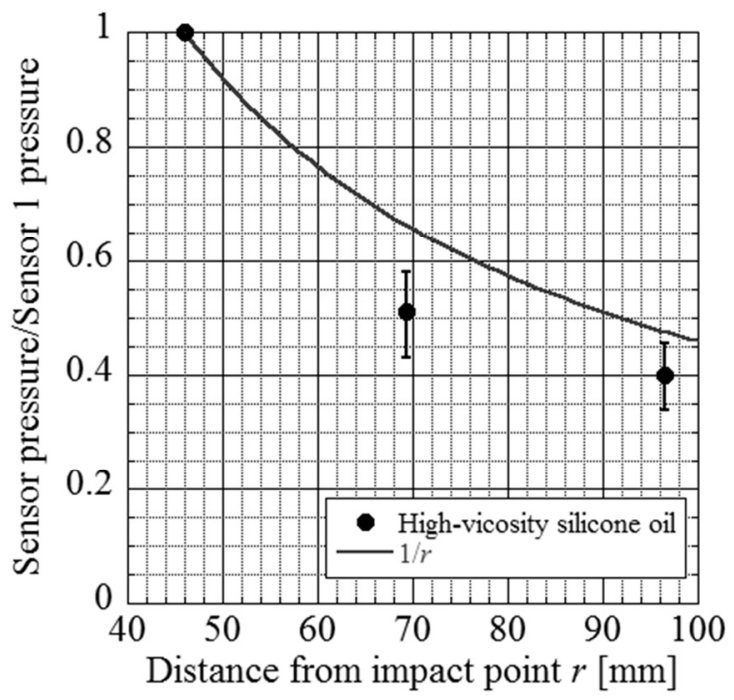

Fig. 13. Comparison between normalized peak pressure for high-viscosity silicone oil and $1 / r$ curve.

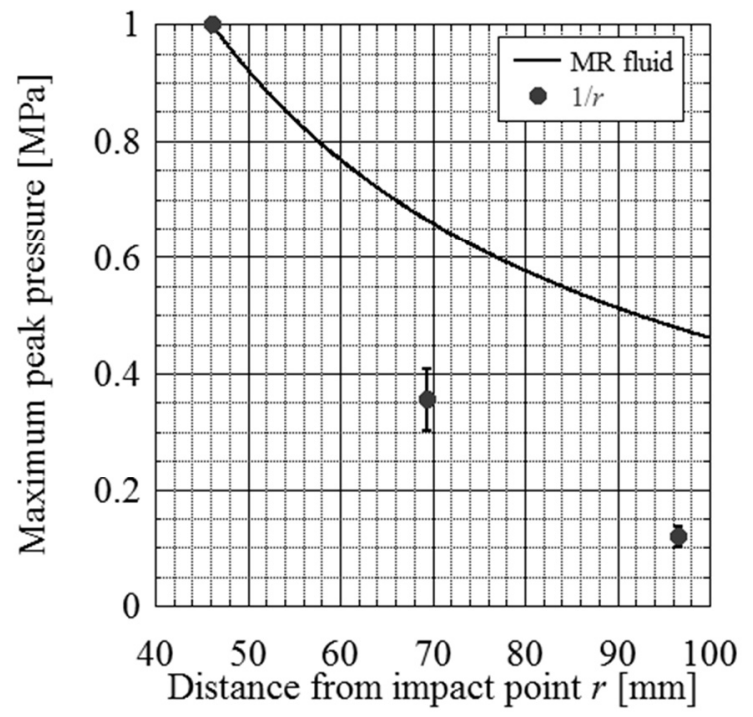

Fig. 14. Comparison between normalized peak pressure for MR fluid and $1 / r$ curve.

\section{Conclusion}

The viscosity of liquids does not affect the deceleration of the projectile. The physical properties of liquids do not affect the attenuation of pressure waves propagating through the fluid. The penetration speed depends on the propagation distance and decelerates at regular intervals. The viscous resistance provided by the liquid does not act on the sides of the projectile. Instead, the viscous resistance presented by air acts on the sides of the projectile. We infer that the projectile speed does not depend on the kinematic viscosity of the liquid. The compressibility affects the maximum value of the pressure wave. As the propagation distance increases, the pressure wave is attenuated. However, the attenuation trend is different for different liquids. We conclude that the attenuation trend is affected by kinematic viscosity, sound speed, and density.

\section{References}

1. J.D. Carlson, M.R. Jolly, Mechatronics 10, 555569 (2000)

2. S.A. Mazlan, N.B. Ekreem, A.G. Olabi, J. Mater. Process. Technol. 201, 780-785 (2008)

3. Y. Nahmad-Molinari, C.A. Arancidia-Bulnes, J.C. Ruiz-Suarez, Phys. Rev. Lett. 82, (1999)

4. W. Huang, W. Zhang, P. Ren, Z. T. Guo, N. Ye, D. C. Li, Y. B. Gao, Exp. Mech. 55, 1123-1138 (2015) 
